\title{
COLINA E BETAÍNA EM RAÇÕES PURIFICADAS NA NUTRIÇÃO DA TILÁPIA DO NILO (OREOCHROMIS NILOTICUS) ${ }^{1}$
}

\author{
Ivan Vieira ${ }^{2,5}$; José Eurico Possebon Cyrino ${ }^{3 *}$; Luiz Edivaldo Pezzato ${ }^{4}$ \\ ${ }^{2}$ Pós-Graduando em Ciência Animal e Pastagens - USP/ESALQ. \\ ${ }^{3}$ Depto. de Produção Animal - USP/ESALQ, C.P. 9 - CEP: 13418-900 - Piracicaba, SP. \\ ${ }^{4}$ Depto. de Melhoramento e Nutrição Animal, Lab. de Nutrição de Organismos Aquáticos - CAUNESP/ FMVZ/UNESP, \\ C.P. 560 - CEP: 18618-000 - Botucatu, SP. \\ ${ }^{5}$ Bolsista CNPq. \\ *Autor correspondente <jepcyrin@carpa.ciagri.usp.br>
}

RESUMO: Problemas metabólicos observados em produções intensivas de tilápias do Nilo (Oreochromis niloticus) têm sido relacionados à deficiência de colina nas rações. Com o objetivo de avaliar o efeito da suplementação dietética da colina na nutrição da espécie, rações purificadas contendo 0; 375; 750; 1.125; 1.500 ou 1.875 mg de cloreto de colina por kg, foram administradas ad libitum por 42 dias a tilápias do Nilo $(5,09 \pm 0,14 \mathrm{~g})$, estocados em gaiolas de PVC atóxico (volume $=60 \mathrm{~L}$ ), alojadas em caixas de polipropileno de $1000 \mathrm{~L}$, em ambiente com condições controladas de temperatura e luminosidade, num delineamento experimental em blocos incompletos casualizados, com três parcelas por bloco $(n=5)$. O ganho de peso (GDP) e o índice de conversão alimentar (ICA) de todos os tratamentos foram superiores ao controle. Não foram observadas diferenças para a quantidade de lipídios no fígado e tecido corporal, e sobrevivência (S\%). Num segundo experimento, os peixes foram alimentados com rações suplementadas com 1.250 ou 2.500 mg de cloreto de colina por kg; ou 1.000; 2.000 ou $3.000 \mathrm{mg}$ de betaína por $\mathrm{kg}$. Não foram observadas diferenças significativas para $\mathrm{S} \%$ e acúmulo de lipídeos hepáticos ou corporais; o ICA e GDP dos tratamentos suplementados com colina foram superiores aos dos tratamentos suplementados com betaína, mas não diferiram entre si. Níveis de suplementação superiores a $375 \mathrm{mg}$ de cloreto de colina por kg de alimento melhoram o ICA e o GDP da tilápia do Nilo, mas a betaína não substitui efetivamente a colina em rações para a espécie.

Palavras-chave: ciclídeos, nutrição, fator lipotrófico, lecitina, lipídios hepáticos

\section{CHOLINE AND BETAINE IN PURIFIED DIETS FOR NILE TILAPIA (OREOCHROMIS NILOTICUS)}

\begin{abstract}
Metabolic problems detected in intensively raised Nile tilapia (Oreochromis niloticus) are credited to possible sub-supplementation of coline in commercial feeds. To investigate the utilization of choline and betaine as feed supplement for the Nile tilapia, groups of 10 fingerlings $(5.09 \pm 0.14 \mathrm{~g})$ stocked in $30 \mathrm{PVC}$ cages $(60 \mathrm{~L}$ ), kept under controlled environmental conditions inside 1000-L plastic pools, were fed to satiation, for 42 days, with purified diets supplemented with $0,375,750,1125,1500$ or $1875 \mathrm{mg}$ of choline chloride per $\mathrm{kg}$ of feed. Trial was set up in an incomplete, totally randomized block design $(\mathrm{n}=5)$. There were no significant differences among treatments for total body and liver lipid contents, and survival rate (S\%); there were significant differences for weight gain (WG) and food conversion ratio (FCR) between the control and all the other treatments, but not between supplementation levels. A second trial (52 days) evaluated the effects of higher rates of choline -1250 or $2500 \mathrm{mg}$ per $\mathrm{kg}$ of feed, and the substitution of choline by betaine in the diets 1000,2000 or $3000 \mathrm{mg}$ of betaine per $\mathrm{kg}$ of feed. There were no significant differences among treatments for total body and liver lipid contents, and S\%, but the FCR and WG of fish fed betaine-supplemented and control diets were significantly worse. There were no differences on the performance among groups fed the cholinesupplemented diets. Supplementation of $375 \mathrm{mg}$ of choline per $\mathrm{kg}$ of feed met nutritional requirements of Nile tilapia, and betaine did not substitute choline effectively in diets for the species.
\end{abstract}

Key words: cichid fish, nutrition, lipotrophic factor, lecithin, hepatic lipids

\section{INTRODUÇÃO}

Considerando a importância da piscicultura para a agro-indústria brasileira, a realização de trabalhos relativos à determinação de exigências nutricionais de espécies com cadeia agro-industrial bem definida é uma necessidade imediata. Neste contexto a tilápia do
Nilo (Oreochromis niloticus), é uma espécie reconhecidamente importante para a aquicultura brasileira em função do grande potencial de produção que apresenta.

A produção mundial de tilápias cresceu de 515.000 toneladas métricas em 1984 para 1,16 milhão em 1995 , sendo que a aquicultura contribuiu com $38 \%$ e $57 \%$, 
respectivamente, deste total. A tilápia do Nilo responde por $72 \%$ do total produzido pela aquicultura, ou seja 474.000 toneladas métricas (Rana, 2000). As tilápias do Nilo, moçambicana e áurea pertencem todas a um mesmo grupo de espécie indistintamente chamadas de tilápias, grupo este que representou $99,5 \%$ do total de ciclídeos produzidos mundialmente em 1995 (Rana, 2000).

A colina ou fator lipotrófico, encontrada tanto em células animais como vegetais, pode se apresentar de três formas: colina livre; acetilcolina; ou lecitina em fosfolipídeos. As funções básicas da colina no metabolismo animal são: componente essencial da acetilcolina, um neurotransmissor do qual a colina é precursor; fosfatidilcolina, que é um elemento estrutural da membrana celular, na transmissão do impulso nervoso e também na utilização de lipídeos; e precursor da betaína, um doador de grupos metil para as reações de metilação e formação da metionina.

Existe uma variação muito grande em relação às exigências de colina por várias espécies de crustáceos, variando de 0,6 até $3 \mathrm{~g} \mathrm{~kg}^{-1}$. Entretando, Teshima et al., (1993) encontraram dados que permitem discutir a necessidade ou não da inclusão de colina em rações para camarões.

As exigências de colina em peixes variam em função da espécie, da fase de desenvolvimento, e também em relação aos parâmetros utilizados para avaliação, como ganho de peso, concentração de lipídios no fígado e tecido corporal. Woodward (1994) reporta que as exigências nutricionais de colina para salmonídeos podem variar de 830 a $4050 \mathrm{mg} \mathrm{kg}^{-1}$.

Analisando o crescimento, a eficiência alimentar, a concentração total de lipídios no plasma e fígado, as concentrações plasmáticas de triglicerídeos, colesterol e fosfatidilcolina de "red drum" (Sciaenops ocellatus), alimentados com rações contendo 250, 500, 750, 1000 e 1500 mg de colina, e uma ração padrão (sem inclusão desse nutriente), Craig \& Gatlin (1996) determinaram que as exigências em colina da espécie variam entre 330$676 \mathrm{mg} \mathrm{kg}^{-1}$.

Griffin et al. (1994), utilizando duas fontes de colina, bitrato de colina e cloreto de colina, em níveis de $0,250,500,1.000,2.000,4.000,6.000$ e $8.000 \mathrm{mg}$ na alimentação de juvenis do híbrido "sunshine bass" (Morone saxatilis $X$ M. chrysops), observaram que a inclusão de $500 \mathrm{mg} \mathrm{kg}^{-1}$ de cloreto de colina na ração proporciona as melhores respostas em termos de ganho de peso e concentração de lipídios no fígado da espécie.

A betaína é um composto aromático encontrado naturalmente nas células animais. Atua na regulação do equilíbrio osmótico, sendo a única fonte doadora de grupos metil prontamente ativa. Outros doadores, como a colina e a metionina, necessitam passar por transformações, para serem utilizados pelos animais: a colina deve ser convertida em betaína na mitocondria celular e a metionina precisa ser ativada através da síntese de S-adenosil-metionina (SAM) (Betancourt, 1999).
O papel de doador de grupos metil e palatabilizante desempenhado pela betaína foi estudado por Penãflorida \& Virtanen (1996) em juvenis de Penaeus monodon, alimentados com rações contendo alta quantidade de proteína de origem vegetal, com níveis de inclusão de $0 ; 5 ; 10 ; 15$ e $20 \mathrm{~g} \mathrm{~kg}^{-1}$ do produto comercial FinnStim na ração, que possui além da betaína, alanina, isoleucina, leucina, valina e glicina. Esses autores concluíram que o produto é uma alternativa competitiva para balancear rações com grandes quantidades de ingredientes vegetais, as quais poderiam apresentar problemas na qualidade da proteína e desequilíbrio em aminoácidos. O produto adicionado, além de melhorar a palatabilidade das rações, auxiliou a manutenção do equilíbrio osmótico pelos camarões.

Kubitza et al. (1997) demonstraram que a betaína não é eficiente como palatabilizante em rações para largemouth bass (Micropterus salmoides), quando utilizado em rações com altas quantidades de proteína de origem vegetal $\left(0 \mathrm{~g}\right.$ farinha de peixe, $600 \mathrm{~g} \mathrm{~kg}^{-1}$ de farelo de soja na ração). Estes resultados são comparáveis àqueles de Moreau \& Dabrowski (1996), onde a utilização de betaína (20 g kg-1 na ração) como palatabilizante em rações de juvenis de esturjão do lago (Acipenser fulvesens), teve efeito significativo, para sobrevivência e ganho de peso, apenas quando combinada com 30 ou $60 \mathrm{~g} \mathrm{~kg}^{-1}$ de farelo de krill ou concentrado protéico de peixe.

O objetivo deste trabalho foi avaliar a colina na nutrição da tilápia do Nilo (Oreochromis niloticus), e a possibilidade de se utilizar a betaína em substituição à colina em dietas para a espécies.

\section{MATERIAL E MÉTODOS}

Os experimentos foram realizados no Laboratório de Nutrição de Peixes do Setor de Piscicultura do Departamento de Produção Animal, da Escola Superior de Agricultura "Luiz de Queiroz", da Universidade de São Paulo, em Piracicaba, SP.

Os parâmetros oxigênio dissolvido e a temperatura da água do sistema foram monitorados diariamente ( $8 \mathrm{~h} 00$ e 18h00). Semanalmente, eram monitorados os parâmetros de $\mathrm{pH}$, alcalinidade, dureza e os níveis de amônia não ionizada na água.

As rações utilizadas neste estudo, em ambos os experimentos, apresentavam a mesma composição básica e suplementação vitamínica e mineral (TABELAS 1 e 2). O procedimento de preparo das rações seguiu as recomendações de Halver (1991) e do NRC (1993).

Foram utilizados dois lotes monosexo de tilápias do Nilo, obtidos por processo de reversão sexual com utilização do hormônio metil-testosterona, conforme descrito por Popma \& Green (1990). A taxa de reversão sexual dos lotes foi determinada pela técnica do acetocarmin, conforme Guerrero \& Shelton (1974). A porcentagem de machos no lote destinado ao primeiro 
TABELA 1 - Composição centesimal da ração purificada utilizada nos experimentos.

\begin{tabular}{lc}
\hline Ingre diente & Porcentual \\
\hline Albumina & 32,10 \\
Gelatina & 7,70 \\
Amido & 44,13 \\
Óleo de Soja & 6,00 \\
$\alpha$-Celulose & 6,00 \\
Fosfato bicálcico & 3,00 \\
Pré-mistura de vitaminas e minerais & 0,50 \\
Vitamina C & 0,05 \\
Cloreto de sódio & 0,50 \\
BHT & 0,02 \\
\hline Total & 100,00 \\
\hline
\end{tabular}

Níveis de garantia da pré-mistura de vitaminas e minerais (Supremais) por kg de produto: vit. $A=1.200 .000 \mathrm{UI}$; vit. $\mathrm{D} 3=200.000$ UI; vit. E $12.000 \mathrm{mg}$; vit. $K 3=2.400 \mathrm{mg}$; vit. $B_{1}=4.800 \mathrm{mg}$; vit. $B_{2}=4.800$ $\mathrm{mg}$; vit. $B_{6}=4.000 \mathrm{mg}$; vit. $B_{12}=4.800 \mathrm{mg}$; ác. Fólico $=1.200 \mathrm{mg}$; pantotenato de cálcio $=1.200 \mathrm{mg}$; vit. $\mathrm{C}=48.000 \mathrm{mg}$; biotina $=48$ $\mathrm{mg}$; niacina $=24.00 \mathrm{mg}$; ferro $=10.000 \mathrm{mg}$; cobre $=600 \mathrm{mg}$; manganês $=4.000 \mathrm{mg}$; zinco $=6.000 \mathrm{mg}$; iodo $=20 \mathrm{mg}$; cobalto $=$ $2 \mathrm{mg}$; selênio $=20 \mathrm{mg}$.

Vit. C (Lutavit C- Aquastab); sal cálcico 2 - monofosfato de ácido ascórbico com $42 \%$ de princípio ativo.

$\mathrm{BHT}=$ Butil hidroxi tolueno

TABELA 2 - Composição bromatológica das rações experimentais.

\begin{tabular}{lcc}
\hline Parâmetro & Ração a mostra 1 & Ração amostra 2 \\
\hline Matéria seca & 92,30 & 92,84 \\
Proteína Bruta & 33,67 & 34,67 \\
Extrato etéreo & 4,03 & 4,66 \\
Material mineral & 5,07 & 3,09 \\
Fibra bruta & 5,60 & 6,11 \\
\hline
\end{tabular}

ensaio foi de $93 \%$, e no lote destinado ao segundo ensaio a porcentagem de machos foi de $97 \%$. Estes valores estão dentro dos padrões aceitáveis para taxa de reversão sexual (Popma \& Lovshin, 1996). Antes do início dos experimentos, os peixes passaram por um período de adaptação às instalações e rotina do laboratório de 3 dias.

\section{Experimento I}

Foram utilizados dez exemplares de tilápias do Nilo, com peso médio de $5,09 \pm 0,14 \mathrm{~g}$, estocados em gaiolas de tela de policloreto de vinila átoxico de abertura de malha $5 \mathrm{~mm}$ e volume unitário de $60 \mathrm{~L}$ (dimensões de $52 \times 27 \times 44 \mathrm{~cm}$ ), compondo uma parcela. Os peixes foram alimentados ad libitum, duas vezes ao dia, sete dias por semana, por seis semanas (42 dias), com seis dietas experimentais contendo 0,$0 ; 375 ; 750 ; 1125 ; 1500$ ou $1875 \mathrm{mg}$ de cloreto de colina (na forma líquida, com $75 \%$ de cloreto de colina) por kg de ração, em um delineamento experimental em blocos incompletos totalmente ao acaso, com três parcelas em cada bloco, com cinco repetições por tratamento (Cochran \& Cox, 1957; Steel \& Torrie, 1980). Utilizaram-se dez caixas polipropileno com capacidade unitária de $1000 \mathrm{~L}$, abastecidas por um sistema fechado de recirculação de água, dotado de filtro biológico. O controle de temperatura foi realizado por uma bomba trocadora de calor. O fluxo d'água permitia um mínimo de seis trocas diárias de todo o volume das caixas. $O$ fotoperíodo foi de $12 \times 12$ horas.

Durante o período experimental foram realizadas quatro biometrias: inicial, aos quinze dias, aos trinta dias e ao final do experimento. Foi adotado o seguinte procedimento para a execução das biometrias: todos os exemplares de cada parcela eram capturados e anestesiados por imersão em solução de benzocaína, $\left(0,1 \mathrm{~g} \mathrm{~L}^{-1}\right.$ de água); lotes de cinco peixes eram selecionados ao acaso e pesados em balança eletrônica de precisão $(0,001 \mathrm{~g})$.

Ao final do período experimental, dois exemplares de cada parcela foram coletados ao acaso e triturados em liquidificador. O material coletado foi processado e armazenado para posterior análise do extrato etéreo no tecido hepático e corporal. Os exemplares restantes foram sacrificados e laparotomizados para remoção do tecido hepático, que também foi armazenado para posterior análise. $O$ desempenho dos peixes foi avaliado com base na variação do ganho de peso, sobrevivência, conversão alimentar, quantidade total de lipídios no corpo e no fígado.

\section{Experimento II}

Grupos de dez tilápias do Nilo com peso médio de 2,14 $\pm 0,07 \mathrm{~g}$ foram estocados em 30 gaiolas e foram alimentados ad libitum, duas vezes ao dia, sete dias por semana, durante 52 dias, com seis dietas experimentais contendo 1250 ou $2500 \mathrm{mg} \mathrm{kg}^{-1}$ de cloreto de colina no alimento; 1000,2000 ou $3000 \mathrm{mg} \mathrm{kg}^{-1}$ de betaína no alimento, e uma ração controle, em um delineamento experimental em blocos incompletos totalmente ao acaso, com cinco repetições por tratamento. Os procedimentos adotados para a realização das biometrias, coleta e armazenagem do material biológico para análise, e parâmetros de desempenho e composição corporal e do fígado foram idênticos àqueles do Experimento I.

\section{Procedimentos analíticos}

Para análise de extrato etéreo, no tecido corporal e hepático, foi utilizada a metodologia de lavagem sucessiva da amostra com o solvente éter de petróleo, em aquecedor cibelin e conjunto extrator de Soxhlet. $O$ extrato etéreo era então calculado por método direto, considerando-se o extraído, da seguinte forma:

Extrato etéreo $=p^{\prime}-p$, sendo, $p^{\prime}=$ peso do balão mais extraído, $p=$ tara do balão, $\%$ extrato etéreo $=$ $\left(p^{\prime}-\text { p/peso da amostra }\right)^{*} 100$ 


\section{RESULTADOS E DISCUSSÃO}

A variação dos parâmetros de qualidade da água, monitorados durante a execução dos dois experimentos, foi de: temperatura $28,63 \pm 0,66^{\circ} \mathrm{C}$;

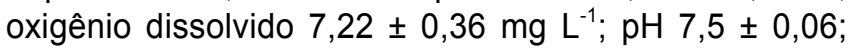
alcalinidade 112,67 $\pm 8,9 \mathrm{mg} \mathrm{CaCO}_{3} \mathrm{~L}^{-1}$; dureza 90,00 \pm $7,1 \mathrm{mg} \mathrm{L}^{-1}$ de $\mathrm{CaCO}_{3}$; amônia total $0,16 \pm 0,08 \mathrm{mg} \mathrm{L}^{-1}$. Esses valores são adequados ao conforto ambiental da espécie em estudo e as variações observadas estão dentro dos intervalos recomendados para a criação de peixes (Boyd, 1996; Keenleyside, 1990).

Não foram observados efeitos dos blocos e diferenças de desempenho e concentração corporal e hepática de lipídios nos peixes do Experimento I (TABELA 3). Com exceção do tratamento com 1125 $\mathrm{mg} \mathrm{kg}^{-1}$ de colina no alimento, onde a sobrevivência foi de $98 \%$, a taxa de sobrevivência dos demais tratamentos foi de $100 \%$.

Foram observadas diferenças significativas para ganho de peso e conversão alimentar apenas entre o tratamento controle e os demais tratamentos, que não diferiram estatisticamente entre si. A suplementação com $375 \mathrm{mg} \mathrm{kg}^{-1}$ de cloreto de colina é suficiente para atender as exigências nutricionais da tilápia para este nutriente.

A não ocorrência de diferenças significativas na concentração de lipídios no fígado e tecido corporal da tilápia nilótica difere dos resultados obtidos por Craig \& Gatlin (1997), que alimentaram juvenis de "red drum" (Sciaenops ocellatus), com peso médio de $2,6 \mathrm{~g}$ com ração semipurificada contendo $3.000 \mathrm{mg} \mathrm{kg}^{-1}$ de colina na ração, na forma de cloreto de colina ou colina bitrato, e observaram um aumento na quantidade de lipídios no músculo e diminuição da quantidade de lipídios no fígado. Entretanto, ao contrário dos resultados aqui observados, a suplementação com colina não teve efeito positivo sobre a conversão alimentar do "red drum".

Diferentemente dos resultados obtidos com a tilápia nilótica, Roem et al. (1990) não observaram diferenças significativas no ganho de peso, conversão alimentar e sobrevivência de tilápias áurea alimentadas com rações purificadas suplementadas com 0; 250; 500; 1.000 ou $2.000 \mathrm{mg}$ de colina por $\mathrm{kg}$ de ração. Esses autores observaram apenas um aumento na quantidade de lipídios no fígado, de $40 \mathrm{~g} \mathrm{~kg}^{-1}$ no início para $80 \mathrm{~g} \mathrm{~kg}^{-1}$ no final do experimento, mas não conseguiram determinar as causas desta variação e consideram não ser necessária a suplementação com colina nas rações para tilápias áurea criadas em sistemas de recirculação de água, provavelmente em função do aproveitamento das bactérias presentes no sistema pela espécie.

O tamanho inicial dos peixes utilizados nos trabalhos com tilápia do Nilo foram superiores a $2 \mathrm{~g}$, motivo provável para as diferenças em relação ao trabalho de Poston (1990a), com salmões do Atlântico (Salmo salar) com pesos inicias de 0,18; 1,0; 1,7 e 7,5 $\mathrm{g}$, alimentados com uma ração semipurificada suplementada com $3 \mathrm{~g} \mathrm{~kg}^{-1}$ de colina, onde ocorreu um aumento no teor de lipídios corporais somente para peixes com peso inicial inferior a $1,7 \mathrm{~g}$ e a suplementação alimentar com colina não afetou a conversão alimentar do salmão do Atlântico.

Trabalhando com alevinos de truta arco-íris (Oncorhynchus mykiss) com peso inicial entre 0,10-0,12 g, Poston (1990b) observou que os peixes alimentadas com rações semipurificadas suplementadas com $3 \mathrm{~g} \mathrm{~kg}$ ${ }^{1}$ de colina, apresentaram melhor crescimento em comparação aos não suplementados. Estes resultados são semelhantes àqueles obtidos neste estudo, com tilápia nilótica. Já em relação à taxa de sobrevivência, os exemplares de truta obtiveram um desempenho bastante superior ao controle, porém inferior aos que receberam uma ração com $720 \mathrm{~g} \mathrm{~kg}^{-1}$ de farinha de arenque. Entretanto, os resultados de Poston (1990b) podem estar associados tanto ao maior teor de proteína, como a maior quantidade e melhor balanço de aminoácidos na ração que continha farinha de peixe.

Rumsey (1991) alimentou trutas arco-íris com uma ração purificada contendo (a) diferentes níveis de inclusão de colina $(0 ; 200 ; 400 ; 600 ; 800$ ou 1.000 $\mathrm{mg} \mathrm{kg}^{-1}$ ), (b) um inibidor da síntese de colina, o 2-amino2-metil-1-propanol (AMP), incluído na dose de $3 \mathrm{~g} \mathrm{~kg}^{-1}$; (c) $3 \mathrm{~g} \mathrm{~kg}^{-1}$ de AMP + $1.000 \mathrm{mg} \mathrm{kg}^{-1}$ de colina; ou (d) uma ração suplementada com $3,7 \mathrm{~g} \mathrm{~kg}^{-1}$ de metionina, concluindo que a suplementação alimentar com colina se mostrou melhor que os tratamentos com inibidor ou

TABELA 3 - Resultados de desempenho e concentração corporal e hepática de lipídios obtidos no Experimento I.

\begin{tabular}{|c|c|c|c|c|c|c|}
\hline $\begin{array}{l}\text { Suplementação } \\
\text { de colina } \mathrm{kg}^{-1} \mathrm{de} \\
\text { alimento }\end{array}$ & $\begin{array}{l}\text { Peso inicial } \\
\text { do lote }\end{array}$ & Ganho de peso & $\begin{array}{l}\text { Conversão } \\
\text { alimentar }\end{array}$ & $\begin{array}{l}\text { Concentração de } \\
\text { lip ídios no tecido } \\
\text { corporal }\end{array}$ & $\begin{array}{l}\text { Concentração } \\
\text { de lipídios no } \\
\text { fígado }\end{array}$ & Sobrevivência \\
\hline $\mathrm{mg}$ & --------------- $g$ & | -----------------_ & $\mathrm{g} \mathrm{g}^{-1}$ & ---------- g 100 & $g^{-1}$ & $\%$ \\
\hline 0 & $50,99 \pm 1,24$ & $97,43 \pm 5,23$ & $1,37 \pm 0,11$ & $20,92 \pm 1,43$ & $5,74 \pm 0,92$ & $100 \pm 0,00$ \\
\hline 375 & $50,18 \pm 0,23$ & $108,12 \pm 3,75$ & $1,21 \pm 0,05$ & $20,72 \pm 1,43$ & $5,70 \pm 0,76$ & $100 \pm 0,00$ \\
\hline 750 & $52,09 \pm 2,52$ & $104,86 \pm 3,40$ & $1,24 \pm 0,05$ & $21,56 \pm 1,54$ & $4,82 \pm 0,84$ & $100 \pm 0,00$ \\
\hline 1125 & $51,31 \pm 1,41$ & $106,63 \pm 8,97$ & $1,29 \pm 0,13$ & $20,94 \pm 0,93$ & $6,84 \pm 0,91$ & $98 \pm 4,47$ \\
\hline 1500 & $50,04 \pm 0,38$ & $105,32 \pm 3,93$ & $1,26 \pm 0,04$ & $21,54 \pm 0,47$ & $5,00 \pm 2,65$ & $100 \pm 0,00$ \\
\hline 1875 & $50,60 \pm 0,54$ & $105,11 \pm 4,21$ & $1,22 \pm 0,03$ & $21,21 \pm 1,82$ & $6,16 \pm 1,71$ & $100 \pm 0,00$ \\
\hline
\end{tabular}

Scientia Agricola, v.58, n.4, p.675-680, out./dez. 2001 
com suplementação de metionina em relação à conversão alimentar. A adição do AMP não teve efeito quando foi utilizada a suplementação com colina. Embora o melhor resultado para ganho de peso tenha sido observado para um valor estimado de $813 \mathrm{mg} \mathrm{kg}^{-1}$ de colina, todos os resultados não diferiram entre si quando o nível de suplementação foi superior a $200 \mathrm{mg} \mathrm{kg}^{-1}$. Estes resultados são semelhante àqueles obtidos neste estudo (TABELA 4) com tilápia nilótica, em que a suplementação com $350 \mathrm{mg} \mathrm{kg}^{-1} \mathrm{de}$ cloreto de colina atendeu as exigências nutricionais da tilápia.

Não foi observada influência dos blocos no desempenho dos peixes. Para análise e comparação dos resultados de conversão alimentar, ganho de peso, sobrevivência, concentração corporal e hepática de lipídios e de extrato etéreo no fígado e no corpo total dos diferentes tratamentos, foram utilizados contrastes, assim definidos: $Y 1=$ tratamento controle vs. demais tratamentos; $Y 2=$ doses de colina vs. doses de betaína; $Y 3$ = entre as doses de betaína; Y4 = efeito linear das doses de betaína; $Y 5=$ efeito quadrático das doses de betaína; $\mathrm{Y} 6=$ entre as doses de colina.

Não foram observadas diferenças entre os tratamentos para nenhum dos contrastes testados para teores de lipídios corporais e hepáticos e sobrevivência. Para o contraste Y6 também não foram detectadas diferenças significativas para os parâmetros de ganho de peso e conversão alimentar.

No contraste $Y 1$ foi constatada diferença para ganho de peso e conversão alimentar. No contraste Y2, ocorreram diferenças significativas entre colina e betaína, tanto para ganho de peso como para conversão alimentar, demostrando também um efeito superior da colina sobre a betaína.

$\mathrm{O}$ contraste $\mathrm{Y} 3$ revela diferenças entre as doses de betaína. Correlacionando-se com os contrastes Y4 (efeito linear) e Y5 (efeito quadrático), a equação linear foi a que melhor se adequou aos resultados. As equações que melhor expressam o efeito negativo da adição de maiores quantidades de betaína nas dietas, para o intervalo utilizado, são as seguintes:
Conversão alimentar

Ganho de peso

$$
\begin{array}{r}
Y=-3,52+0,003844 X \\
Y=130,18-0,034725 X
\end{array}
$$

Hughes (1993) utilizou diversos estimulantes para aumentar o consumo de ração e observou menor consumo de alimento por alevinos de salmão Chinook alimentadas com ração suplementada com

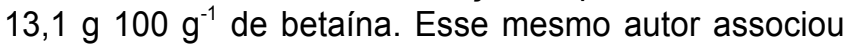
essa diminuição do consumo à forma da betaína adicionada, que foi a hidrocloreto.

Rumsey (1991) observou que alevinos de trutas alimentadas com ração suplementada com betaína apresentaram desempenho inferior àquelas truta alimentadas com ração suplementada com colina, em relação ao ganho de peso e conversão alimentar. Esse mesmo autor observou que embora a suplementação com betaína não eliminasse o efeito negativo da adição de AMP às rações, o desempenho dos peixes alimentados com rações suplementadas com betaína foi superior àquele dos peixes que receberam rações suplementadas com metionina.

Em todos os ensaios conduzidos por Rumsey (1991) não foram constatadas variações nos teores de lipídios hepáticos das trutas. Estes resultados são semelhantes àqueles obtidos neste estudo com a tilápia nilótica, onde também não foram observadas variações significativas na concentrações de lipídios hepáticos ou corporais. O desempenho dos peixes que receberam rações suplementadas com betaína foi inferior tanto para ganho de peso como conversão alimentar, em comparação aos tratamentos com colina, assim como os peixes alimentados com a ração suplementada com $3.000 \mathrm{mg} \mathrm{kg}^{-1}$ de betaína na ração apresentaram desempenho inferior aos peixes que receberam a ração controle.

Os peixes alimentados com as rações suplementadas com betaína apresentaram comportamento apático, demorando um tempo superior aos demais tratamentos para iniciar a captura do alimento. Isso pode ter sido resultante de um efeito negativo da suplementação dietética com betaína, tanto sobre a palatabilidade das rações, como sobre as rotas metabólicas dos peixes.

\begin{tabular}{|c|c|c|c|c|c|c|}
\hline $\begin{array}{l}\text { Suplementação } \\
\text { de colina } \mathrm{kg}^{-1} \\
\text { de alimento }\end{array}$ & $\begin{array}{c}\text { Peso inicial } \\
\text { do lote }\end{array}$ & $\begin{array}{c}\text { Ganho de } \\
\text { peso }\end{array}$ & $\begin{array}{c}\text { Conversão } \\
\text { alimentar }\end{array}$ & $\begin{array}{l}\text { Concentração de } \\
\text { lip ídios no tecido } \\
\text { corporal }\end{array}$ & $\begin{array}{l}\text { Concentração } \\
\text { de lipídios no } \\
\text { fígado }\end{array}$ & Sobrevivência \\
\hline $\mathrm{mg}$ & \multicolumn{2}{|c|}{ 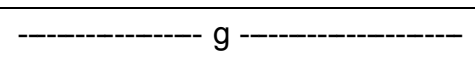 } & $\mathrm{g} \mathrm{g}^{-1}$ & \multicolumn{2}{|c|}{---------- g 100 g $^{-1}$------------ } & $\%$ \\
\hline 0 & $21,90 \pm 0,66$ & $87,22 \pm 4,5$ & $1,64 \pm 0,11$ & $27,90 \pm 2,45$ & $10,66 \pm 2,09$ & $98 \pm 4,47$ \\
\hline colina, 1250 & $21,49 \pm 0,66$ & $89,09 \pm 8,19$ & $1,57 \pm 0,15$ & $28,73 \pm 2,08$ & $11,58 \pm 3,57$ & $94 \pm 9,94$ \\
\hline colina, 2500 & $21,32 \pm 0,86$ & $93,03 \pm 6,35$ & $1,52 \pm 0,10$ & $28,93 \pm 0,93$ & $9,70 \pm 1,47$ & $100 \pm 0,00$ \\
\hline betaina, 1000 & $21,44 \pm 0,66$ & $81,96 \pm 5,93$ & $1,61 \pm 0,14$ & $27,17 \pm 1,95$ & $8,45 \pm 0,57$ & $96 \pm 5,48$ \\
\hline betaína, 2000 & $21,88 \pm 0,87$ & $87,72 \pm 12,11$ & $1,60 \pm 0,22$ & $27,26 \pm 1,36$ & $12,01 \pm 2,66$ & $94 \pm 5,48$ \\
\hline betaína, 3000 & $21,39 \pm 0,83$ & $12,51 \pm 2,83$ & $9,30 \pm 2,05$ & $28,68 \pm 1,78$ & $13,92 \pm 2,32$ & $94 \pm 8,94$ \\
\hline
\end{tabular}

TABELA 4 - Resultados de desempenho e concentração corporal e hepática de lipídios obtidos no Experimento II.

Scientia Agricola, v.58, n.4, p.675-680, out./dez. 2001 


\section{CONCLUSÕES}

As exigências nutricionais de colina para alevinos de tilápia do Nilo (Oreochromis niloticus) podem ser satisfeitas com a adição de $375 \mathrm{mg}$ de cloreto de colina por $\mathrm{kg}$ de alimento. A suplementação com betaína não poderia substituir a suplementação com colina em rações para a tilápia do Nilo.

\section{REFERÊNCIAS BIBLIOGRÁFICAS}

BETANCOURT, R. Betafin ${ }^{\circledR}$ conceitos básicos e novas aplicações na nutrição de suínos. s.l., 1999.

BOYD, C.E. Water quality in ponds for aquaculture. Songkhala: Shrimp Mart, 1996. 482p.

COCHRAN, W.G.; COX, G.M. Experimental designs. 2.ed. New York: John Wiley \& Sons, 1957. 614p.

CRAIG, S.R.; GATLIN III, D.M. Dietary choline requirement of juvenile red drum (Sciaenops ocellatus). The Journal of Nutrition, v.126, p.1696-1700, 1996.

CRAIG, S.R.; GATLIN III, D.M. Growth and body composition of juvenile red drum (Sciaenops ocellatus) fed diets containing lecithin and supplemental choline. Aquaculture, v.151, p.259267, 1997.

GUERRERO, R.D.; SHELTON, W.L. An aceto-carmine squash method for sexing juvenile fishes. The Progressive Fish Culturist, v.36, p.56, 1974.

GRIFFIN, M.E.; WILSON, K.A.; WHITE, M.R.; BROWN, P.B. Detary choline requirement of juvenile hybrid striped bass. The Journal of Nutrition, v.124, p.1685-1689, 1994.

HALVER, J.E. The vitamins. In: HALVER, J.E. (Ed.) Fish nutrition. San Diego: Academic Press, 1991. p.32-111.

HUGHES, S.G. Single-feeding response of chinook salmon fry to potential feed intake modifiers. The Progressive Fish Culturist, v.55, p.40-42, 1993.

KEENLEYSIDE, M.H.A. Cichlid fishes: Behaviour, ecology and evolution. London: Chapman \& Hall, 1990. 378p. (Fish and Fisheries Series, 2).

KUBITZA, F.; LOVSHIN, L.L.; LOVELL, R.T. Identification of feed enhancers for juvenile largemouth bass Micropterus salmoides. Aquaculture, v.148, p.191-200, 1997.

MOREAU, R.; DABROWSKI, K. Feeding stimulants in semipurified diets for juvenile lake sturgeon, Acipenser fulvescens Rafinesque. Aquaculture Research, v.27, p.953957, 1996.
NATIONAL RESEARCH COUNCIL. Nutrient requirements of fish. Washington: National Academy Press, 1993. 114p.

PENÃFLORIDA, V.D.Y.; VIRTANEN, E. Growth, survival and feed conversion of juvenile shrimp (Penaeus monodon) fed a betaine/amino acid additive. The Israeli Journal of Aquaculture, v.48, p.3-9, 1996.

POPMA, T.J.; GREEN, B.W. Sex reversal of tilapia in earthen ponds. Auburn: Auburn International Center of Aquaculture, Alabama Agricultural Experiment Station; Auburn University, 1990. 15p. (Research and Development Series, 35)

POPMA, T.J.; LOVSHIN, L.L. Worldwide prospects for commercial production of tilapia. Auburn: Auburn International Center of Aquaculture, Alabama Agricultural Experiment Station; Auburn University, 1996. 23p. (Research and Development Series, 41)

POSTON, H.A. Effect of body size on growth, survival and chemical composition of Atlantic salmon fed soy lecithin and choline. The Progressive Fish Culturist, v.52, p.226-230, 1990a.

POSTON, H.A. Performance of rainbow trout fry fed supplemental soy lecithin and choline. The Progressive Fish Culturist, v.52, p.218-225, 1990b.

RANA, K.J. Aquatic environments and use of species groups. reported use of culture environments for aquaculture production. http://www.fao.org/fi/publ/ circular/c886.1/ species.asp. (05/04/2000)

ROEM,A.J.; STICKNEY, R.R.; KOHLER, C.C. Vitamin requirements of blue tilapia in recirculating water system. The Progressive Fish Culturist, v.52, p.15-18, 1990.

RUMSEY, G.L. Choline-betaine requirements of rainbow trout (Oncorhynchus mykiss). Aquaculture, v.95, p.107-116, 1991.

STEEL, R.G.D.; TORRIE, J.H. Principles and procedures of statistics: A biometrical approach. New York: McGraw-Hill Book, 1980. 633p.

TESHIMA, S.; KANAZAWA, A.; KOSHIO, S. Recent development in nutrition and microparticulate diets of larval prawns. The Israeli Journal of Aquaculture, v.45, p.175-184, 1993.

WOODWARD, B. Dietary vitamin requirements of cultured young fish, with emphasis on quantitative estimates for salmonids. Aquaculture, v.124, p.133-168, 1994.

$\overline{\text { Recebido em } 30.08 .00}$ 